\title{
Procesamiento cognitivo del Feedback Correctivo Escrito indirecto en los errores preposicionales en ELE
}

\author{
Anita Ferreira Cabrera ${ }^{1}$ \\ Universidad de Concepción, Chile \\ René Oportus Torres ${ }^{2}$ \\ Universidad de Concepción, Chile
}

\begin{abstract}
Resumen
Este estudio muestra los resultados de un experimento cognitivo en aprendientes de Español como Lengua Extranjera (ELE), cuyo objetivo es examinar empíricamente el $\mathrm{rt}$ de procesamiento y la efectividad del Feedback Correctivo Metalingüístico Indirecto (FCMI), comparado con el Feedback Correctivo Metalingüístico Directo (FCMD) durante el tratamiento de los errores preposicionales de $a$, de, por, para y con en aprendientes de ELE de nivel A2 y B1. Los resultados muestran que en el tratamiento de los errores preposicionales el FCMI es el que genera mayor rt de procesamiento tanto en el nivel A2, como en el nivel B1. De igual forma, el FCMI suscitaría una mayor influencia de las diferencias individuales en el
\end{abstract}

\footnotetext{
1 Para correspondencia, dirigirse a: Anita Ferreira Cabrera (aferreir@udec.cl), casilla 160-C, Correo 3, Universidad de Concepción.

2 Para correspondencia, dirigirse a: René Oportus Torres (reneoportus@gmail.com), casilla 160-C, Correo 3, Universidad de Concepción.
} 
procesamiento del FCE que la del FCMD, y esta sería mayor en el nivel B1 que en el nivel A2.

Palabras clave: procesamiento cognitivo del feedback correctivo escrito, feedback correctivo indirecto, tiempos de respuesta (rt), preposiciones por y para.

Cognitive processing of Indirect Written Corrective

FEEDBACK (IWCF) OF PREPOSITIONAL ERRORS IN SPANISH AS A Foreign LANGUAGe (SFL)

\begin{abstract}
This study presents the results of a cognitive experiment in learners of SFL whose objective is to empirically examine processing $\mathrm{rt}$ and effectiveness of Indirect Written Corrective Feedback (IWCF), compared to Direct Written Corrective Feedback (DWCF) during the treatment of prepositional errors of de, por, para and con in SFL learners of levels $\mathrm{A} 2$ and $\mathrm{B} 1$. The results indicate that in the treatment of prepositional errors, it is the IWCF the one that generates higher processing rt both in level A2 and level B1. Likewise, IWCF would exert a greater influence of individual differences in written corrective feedback processing than DWCF, and this would also be greater in level B1 than in level A2.
\end{abstract}

Keywords: cognitive processing of written corrective feedback, indirect written corrective feedback, response time (rt), prepositions por and para.

Recibido: 30/08/17

Aceptado: 30/04/18

\title{
1. INTRODUCCIÓN ${ }^{3}$
}

El estudio que se presenta a continuación forma parte de la investigación sobre el Feedback Correctivo Escrito (FCE) metalingüístico indirecto y su efectividad en el contexto del Proyecto Fondecyt 1140651. La investigación

3 El estudio presentado en este artículo se ha desarrollado en el contexto del proyecto de investigación CONICYT-FONDECYT No. 1140651 "El Feedback Correctivo Escrito Directo e Indirecto en la adquisición y aprendizaje del Español como Lengua Extranjera", cuya Investigadora Responsable es la Dra. Anita Ferreira Cabrera. 
se enmarca dentro del ámbito de la adquisición del Español como Lengua Extranjera (ELE) desde una perspectiva cognitiva, y hace aportes interesantes acerca del procesamiento del FCE durante el tratamiento de errores preposicionales bajo el paradigma del tiempo de respuesta (rt.'response time') (Jiang, 2012).

Las investigaciones sobre el Feedback Correctivo Escrito (FCE) Metalingüístico (Ellis, 2008; Sheen, 2011) han examinado los efectos de recibir versus no recibir estrategias en el corto y largo plazo. El FCE ayuda a desarrollar la conciencia metalingüística a través de una atención selectiva de errores enfocados en el proceso de escritura con el objeto de mejorar la precisión lingüística. Se trata de elevar el nivel de conciencia lingüística de los aprendices, llamándoles la atención sobre sus errores recurrentes, a través de información metalingüística clave para que noten o perciban sus problemáticas y mejoren la comprensión de ellas, optimizando así su habilidad escrita.

Nuestro enfoque de investigación se aboca a estudiar el procesamiento del FCMD y FCMI bajo el paradigma del rt. Conforme a ello, el tiempo que los participantes toman en responder los reactivos experimentales tratados con FCE nos dará luces sobre la carga del procesamiento que ambas modalidades de FCE conllevan. Nuestro interés principal es recopilar datos empíricos más robustos sobre el procesamiento del FCMI en comparación con el del FCMD que sustenten la tesis del mayor esfuerzo y profundidad asociados a este tipo de FCE.

Nos interesa específicamente obtener evidencia más consistente sobre las diferencias de procesamiento del Feedback Correctivo Metalingüistico Indirecto (FCMI), respecto del Feedback Correctivo Metalingüistico Directo (FCMD), en dos niveles de competencia (A2, B1) y los errores de cinco preposiciones específicas ( $a$, de, con, para y por). Para ello, a nivel metodológico, tomamos como base el diseño experimental de Ferreira, Oportus y Fuentes (2016) realizando ajustes a los reactivos y al procedimiento original de recopilación de datos con el objetivo de mejorar la calidad de la evidencia observada.

El artículo está organizado de la siguiente forma: en la primera parte incluimos las bases teóricas sobre las que se sustenta el estudio. En ella damos realce a los aspectos descriptivos y empíricos que definen la dificultad, complejidad de uso y complejidad cognitiva que implica el manejo preposicional en el ámbito de ELE. Así también, esbozamos las dos modalidades de FCE objeto de nuestro estudio y explicamos cómo nos podemos acercar a estimar su procesamiento desde la perspectiva del rt. A continuación, describimos el estudio en términos del diseño y metodología de investigación, seguido de los resultados, su relevancia e implicancias en 
relación con los antecedentes disponibles en otros estudios. Hacia el final del artículo, incluimos las conclusiones más relevantes y proyecciones de la investigación.

\section{MARCO TEÓRICO}

\subsection{LA PROBLEMÁTICA PREPOSICIONAL COMO FOCO DE ESTE ESTUDIO}

Este estudio se focaliza en los errores preposicionales de aprendientes de ELE. Nuestro interés por este aspecto lingüístico se basa en consideraciones teóricas y en la evidencia disponible en estudios de corpus sobre este fenómeno. En primer lugar, dentro del marco de la enseñanza comunicativa de segundas lengua y el enfoque de Atención a la Forma (Long 1991), Harley en 1993 (citado por Williams y Evans 2009, 155) plantea, acerca de la selección de los aspectos lingüísticos sobre los cuales intervenir, que algunas formas resultan más eficaces que otras para lograr ganancias importantes en la precisión. Al respecto, el autor señala 4 criterios que se deberían considerar para seleccionar la forma a tratar: (1) que esta no se diferencie de manera evidente entre la L1 y L2. (2) que sea poco regular o poco frecuente en el input. (3) que sea poco importante en la comunicación y que por ello no impida que se lleve a cabo exitosamente. (4) que pueda conducir a una mala interpretación o a un análisis incorrecto. Tomando en cuenta estos puntos, el uso preposicional se ajusta a estos criterios puesto que consideramos: que está determinado por las diferencias entre los valores de uso asignados a las preposiciones en la lengua meta y la lengua materna (Campillos 2014); que las reglas que lo dirigen no son fácilmente sistematizables; que su aparición posee bajo realce, y que no afecta de manera drástica el propósito comunicativo.

En segundo lugar, el uso preposicional en la producción escrita aparece como uno de los rasgos que presenta mayor dificultad a los estudiantes de ELE, observándose una alta frecuencia y concurrencia de desviaciones en su uso (Regueiro 2014; Giraldo 1997). Esta evidencia es reportada por numerosos estudios que han examinado errores de aprendientes y que concluyen que las preposiciones constituyen una de las mayores fuentes de error en el ámbito gramatical (Giraldo 1997; Perea 2007; Leontaridi 2010; Campillos 2014; Elejalde y Ferreira 2016; Ferreira y Elejalde 2017). Como ejemplo, señalamos tres estudios de corpus de aprendientes de diferentes 
orígenes lingüísticos para ilustrar la marcada dificultad preposicional. Ferreira, Elejalde y Vine (2014), en su estudio en estudiantes de nivel A2 y B1, informan que dentro de la categoría de errores gramaticales los que conciernen a las preposiciones alcanzan la segunda frecuencia más alta, con un $25 \%$. Por su parte, en el estudio de Ferreira y Lafleur (2015) en estudiantes de nivel A2 y B1 nos encontramos con que los errores preposicionales alcanzan el $52 \%$ del total de errores gramaticales, en donde $a$, para, por y de muestran las mayores frecuencias de uso y error. En un ámbito similar, el estudio de Ferreira y Elejalde (2017) sobre análisis de errores recurrentes en aprendientes de nivel A2 y B1 informan que la recurrencia de errores preposicionales es del $10 \%$, en tercer lugar después de los errores de ortografía y de concordancia sintáctica.

Por otra parte, el uso preposicional puede considerarse altamente complejo puesto que adicionalmente a las diferencias de significados y valores de uso entre la L1 y L2, los estudiantes deben manejar los rasgos de naturaleza intralingual del español que permiten que las preposiciones tengan una considerable funcionalidad polisémica, lo que significa que una preposición puede adquirir matices semánticos distintos en diferentes contextos léxicos, y que diferentes preposiciones expresen significados próximos en los mismos contextos (Giraldo, 1997). Al respecto, Fernández López (1994) señala que los usos preposicionales en la lengua se han definido de acuerdo a las restricciones paradigmáticas fijadas por el uso lingüístico, las que tienden a aumentar en los casos de rección, locuciones preposicionales y expresiones idiomáticas. En suma, lo anterior nos lleva a sostener que la relativa complejidad lingüística preposicional se proyecta en una marcada complejidad cognitiva al estudiante puesto que debe sopesar en diferentes niveles de análisis los aspectos que definen la precisión preposicional, y que suponemos explica la generación de los numerosos errores que la afectan.

Para ilustrar en términos genéricos la complejidad lingüística de las preposiciones de este estudio (a, para, por, de y con) nos remitimos a varios trabajos a nuestra disposición. Por ejemplo, las preposiciones que presentan las mayores frecuencias de uso concentran el mayor número de errores preposicionales: $a$, de, por, para, en y con, en orden descendiente (Campillos 2014). Luego, al analizar individualmente los antecedentes por preposición, nos encontramos con que la complejidad de la preposición $a$ estaría dada por su alta productividad y multifuncionalidad: expresar movimiento espacial o temporal, antecedente del complemento indirecto, y ocasionalmente del complemento directo; además de "valores finales, causales, distributivos, modales, instrumentales y condicionales" (Pérez Cino 2000, 11). En cuanto a la preposición para, esta expresa destino en su valor espacial, tiempo futuro aproximado en su valor temporal, y finalidad en su uso nocional. Por su parte, 
la preposición por tiene el significado de movimiento con valor espacial, tiempo aproximado, periodicidad o duración con valor temporal y finalidad, entre varios usos nocionales. Respecto de estas dos últimas preposiciones, Garzón (2012) alude a los sentidos próximos de finalidad expresados por para y por, reflejando un alto grado de sinonimia entre ambas. Por ello, en muchos casos pueden aparecer en los mismos contextos con leves matices de diferencia en su significado, fenómeno que las hace especialmente complejas y confusas (Campillos 2014; Lafleur y Ferreira 2016). Luego, la preposición de expresa significados de posesión, pertenencia, cualidad, origen o procedencia, causa, cantidad indeterminada, tiempo y modo (Garzón 2012). No obstante estos numerosos usos, de presentaría una complejidad relativamente baja, con la menor cantidad reportada de errores en los valores generales (temporales, espaciales y nocionales) de las preposiciones estudiadas debido a una mayor diferenciación implícita entre ellos, concentrando los errores en los usos idiomáticos (Fernández López 1994). Por último, la preposición con expresa varios usos nocionales: instrumento, medio, modo, actitud, contenido, compañía, entre otros. Sin embargo, esta preposición ofrecería una baja complejidad, siguiendo a Campillos (2014), quien indica casos restringidos de error explicados por la conmutabilidad con otras preposiciones, por ejemplo, con la preposición de en el complemento del nombre (Serrano 2016).

\subsection{El procesamiento cognitivo del Feedback Correctivo Escrito}

Ante la problemática preposicional en la escritura en ELE, el FCE se convierte en una de las modalidades de enseñanza de atención en la forma que podemos utilizar para atraer la mirada hacia el error en pos de lograr un mejoramiento en la precisión de los estudiantes. El presente trabajo pone su atención en dos modalidades de FCE localizado. La primera de ellas, el FCMD, trata el error entregando al estudiante la forma correcta esperada junto con un comentario de tipo metalingüístico. La segunda, el FCMI, señala solamente un comentario de naturaleza metalingüística sin dar la respuesta correcta. Desde un punto cognitivo, el FCMD implicaría una carga menor que la que ejerce el FCMI puesto que en el caso del segundo es el estudiante el que tiene que examinar el problema lingüístico a un nivel más profundo y de mayor elaboración para resolverlo, comparado con el FCMD (Ferris 2010). Por este argumento se plantea que el FCMI sería más conducente a la adquisición de largo plazo que el FCMD. No obstante, no disponemos de evidencia irrefutable sobre su efectividad en el largo plazo, con algunos estudios que indican que no existirían diferencias entre los efectos de ambos 
tipos de FCE, con otros que muestran una cierta ventaja del FCMI por sobre el FCMD (Bitchener y Ferris, 2012; Ferreira, 2017).

En un estudio previo en torno al Feedback Correctivo Escrito (FCE) y su efectividad llevada a cabo por Ferreira, Oportus y Fuentes (2016) se define observar el efecto de estrategias de FCE en dos instancias de rt. La primera se refiere a los rt de procesamiento de los estímulos contestados correctamente en un primer intento; la segunda corresponde al tiempo de reacción a un mensaje de FCE cuando un participante comete un error y luego lo corrige como resultado del tratamiento de FCE que recibió. El estudio señalado entrega, en primer lugar, datos contundentes sobre la forma en que los $\mathrm{rt}$ de procesamiento de la primera instancia de respuesta difieren de los rt de procesamiento del FCE de la segunda instancia. En este sentido, el FCE favorecería sustantivamente la reducción del tiempo empleado en procesar un estímulo que ha sido respondido incorrectamente en un primer intento. En segundo lugar, respecto de la segunda instancia de rt, dicho trabajo compara el rt de procesamiento de dos tipos de FCE metalingüístico, directo e indirecto, a lo largo de dos niveles de competencia durante el tratamiento de los errores asociados al uso de cinco preposiciones. Respecto de este segundo cometido, no obstante lo interesante que resultan los hallazgos informados respecto de las diferencias observadas a lo largo de los tipos de FCE, los niveles de competencia y preposiciones estudiadas, algunos de los datos aparecen contradictorios y dificultan el poder observar las tendencias de procesamiento cognitivo con claridad.

El trabajo de Ferreira, Oportus y Fuentes (2016) nos permite contar con algunos antecedentes para enfrentar las preguntas de esta investigación. (1) El procesamiento del FCE estaría vinculado tanto a la competencia como a las preposiciones específicas cuyos errores son tratados. De esta forma, en primer lugar, el rt de procesamiento del FCE del nivel B1 sería mayor que el del nivel A2 en el tratamiento de errores de las preposiciones $a$, de y para, mientras que el nivel $\mathrm{A} 2$ procesaría más rápido el $\mathrm{FCE}$ de los errores de en y por. (2) En el análisis del rt por tipo de FCE y competencia, el nivel B1 procesaría el FCMD en mayor tiempo que el nivel A1 en los errores de las preposiciones $a$, de, en, para y por. Por otra parte, en el caso del FCMI, el nivel A2 procesaría en mayor tiempo los errores de en, para y por, mientras que el nivel B1 procesaría en mayor rt los errores de $a$ y de. (3) El nivel A2 mostraría una mayor dispersión que el nivel $\mathrm{B} 1$ en los rt de procesamiento del FCMI, mientras que en el nivel B1 se daría una mayor amplitud de rt de procesamiento del FCMD.

En definitiva, compararemos los rt de procesamiento del FCE de acuerdo a su tipo, nivel de competencia y en relación con las cinco preposiciones incluidas en este estudio. Las preguntas que orientan la investigación son: 
1. ¿El rt de procesamiento y la efectividad del FCE aparecen vinculados al tipo de FCE y a la competencia?

2. ¿Cómo es el comportamiento del rt de procesamiento del FCMI y su efectividad en relación con la competencia y las preposiciones individuales estudiadas, y en comparación con el FCMD?

\section{EL ESTUDIO}

Esta investigación de carácter cognitivo cuenta con un diseño de tipo experimental cuyo objetivo se centra en examinar el comportamiento del rt de procesamiento de dos modalidades de FCE y su efectividad para facilitar la corrección de errores preposicionales en aprendientes de ELE de nivel A2 y B1. Los tipos de Feedback Correctivo estudiados son el FCE metalingüístico directo (FCMD) y el FCE metalingüístico indirecto (FCMI). Siguiendo a Sheen (2011), definimos al primero como aquel que junto con entregar la respuesta correcta, también incorpora una explicación de índole metalingüística que refuerza el mensaje correctivo; a diferencia del segundo, que solo entrega una explicación metalingüística sin hacer explícita la forma correcta esperada. El rt de procesamiento del FCE corresponde al tiempo de respuesta de la instancia en que un participante responde en forma acertada un estímulo que previamente había respondido en forma errónea y que luego corrige como resultado de la aplicación de una de las dos modalidades de FCE estudiadas. Dicho lapso de tiempo se inicia en el momento que se presenta un mensaje de FCE hasta el momento que el sujeto ingresa la respuesta correcta en segunda instancia. Se define como variable independiente el tipo de FCE, y como variable interviniente relativa a los sujetos el nivel de competencia. Por otra parte, las variables dependientes están representadas por el rt de procesamiento del FCE y su efectividad.

\subsection{HIPÓTESIS DE INVESTIGACIÓN}

En consideración a la naturaleza y características del FCMI señaladas por la literatura especializada, respecto de la forma en que este indica a un estudiante cómo realizar la corrección de un error, debería esperarse una mayor ambigüedad, y por lo tanto un mayor lapso de tiempo de respuesta que el del FCMD en la resolución de un error preposicional dado. En cuanto 
a la competencia, la evidencia con la que contamos sugiere que el nivel B1, debido a un mayor conocimiento de los diferentes aspectos formales y semánticos de la lengua, toma más tiempo en estimar la respuesta correcta que el nivel A2. En lo que respecta a las preposiciones individuales, aquellas cuyo uso presenta un mayor número de errores, conllevarían un mayor tiempo de procesamiento que aquellas que presentan menos errores tratados, reflejando el nivel de dificultad que presentan a los estudiantes y la complejidad de sus usos. De acuerdo con estos antecedentes, se enuncian dos hipótesis de investigación:

$\mathrm{H}_{1}$ : El promedio de los rt en el procesamiento del FCMI será mayor que el del FCMD e irá acompañado de una menor efectividad del FCE en ambos niveles de competencia en un corto plazo.

$\mathrm{H}_{2}$ : El promedio de los $\mathrm{rt}$ en el procesamiento del $\mathrm{FCE}$ será mayor en las preposiciones que presentan mayor número de errores que en las preposiciones menos problemáticas.

\section{METODOLOGÍA}

\subsection{Muestra}

La muestra del experimento estuvo conformada por 26 estudiantes de intercambio estudiantil de nivel de pregrado y postgrado que tomaron cursos de ELE en el Programa de Español como Lengua Extranjera, ELE-UdeC ${ }^{4}$ de la Universidad de Concepción durante el primer y segundo semestre del año 2016. Su nivel de competencia fue determinado al comienzo del programa con la Prueba de Multinivel con Fines Específicos Académicos del Programa ELE-UdeC (Ferreira 2016), instrumento alineado con los niveles del Marco Común Europeo de Referencia (Consejo de Europa 2001). El resultado de la prueba situó a 15 estudiantes en el nivel A2 con lenguas maternas: francés (5), inglés (4), alemán (3), lengua persa (1), y holandés (2). Los 11

\footnotetext{
4 Programa de enseñanza de español como lengua extranjera que imparte cursos de español general y académico bajo una metodología de enseñanza comunicativa (Directora del Programa ELE-UdeC, Dra. Anita Ferreira Cabrera, correo electrónico: ele.udec@udec. cl, página web: http://ele.udec.cl/ ).
} 
alumnos restantes se ubicaron en el nivel B1, con lenguas maternas: inglés (4), alemán (3), francés (3), y portugués (1). La edad promedio de los sujetos era de 22,2 años $\left(\mathrm{SD}^{5}=1.16\right)$.

\subsection{ESTÍMULOS Y DISEÑO DEL EXPERIMENTO}

El experimento se basa en el diseño empleado por Ferreira, Oportus y Fuentes $(2016)^{6}$ con una modificación respecto de las preposiciones utilizadas en ese estudio, descartando la preposición de, y reemplazándola por la preposición con. Adoptamos esta decisión en vista de que el FCE es proporcionado en función de la ocurrencia del error, por ello, seleccionamos las preposiciones $a$, de, para y por puesto que presentan altos índices de frecuencia y recurrencia de usos erróneos (Fernández López 1994; (Giraldo 1997; Perea 2007; Leontaridi 2010; Campillos 2014; Ferreira, Elejalde y Vine 2014; Ferreira y Lafleur 2015; Ferreira y Elejalde 2017). En cuanto a la preposición con, la seleccionamos por las razones contrarias, considerando que presenta relativamente bajos niveles de desviaciones, por lo que se integró de manera que funcionase como un control en el experimento y nos permitiese comparar y observar el procesamiento del FCE con mayor claridad.

El experimento incluyó un total de 120 reactivos (24 por preposición, 3 significados preposicionales por preposición, 8 reactivos por significado preposicional). Los significados preposicionales también fueron escogidos en base a un criterio de la dificultad. De esta forma, se seleccionaron tres usos por preposición que mostraron una alta frecuencia de errores en los estudios preposicionales señalados en el párrafo anterior. En definitiva, se incluyeron los siguientes significados preposicionales por preposición: $a$ (fecha, modo, complemento directo de persona); de (modo, tema, tiempo), con (compañía de personas, instrumento, unión de cosas), para (comparación, finalidad, tiempo), y por (lugar, medio, motivo o razón).

Los estímulos consistían en oraciones simples con un espacio en blanco que debía ser completado con una de las preposiciones del estudio. Para ello, los estudiantes escribían su respuesta por teclado y luego presionaban la tecla ENTER para ingresarla. Los estímulos fueron validados en un ciclo

\footnotetext{
$\mathrm{SD}=$ Standard Variation (Variación Estándar).

6 Para ver representación gráfica y detalles complementarios del diseño experimental, revisar dicho estudio.
} 
de tres etapas previas, que incluyó la aplicación de los reactivos a docentes, estudiantes de español y aprendientes de ELE, y que permitió realizar las adecuaciones para corregir los casos ambiguos, por ejemplo, aquellos que podían haber aceptado más de una respuesta.

\subsection{Procedimientos}

El experimento fue desarrollado con el software Psychology Software Tools E-Prime 2.0 (Schneider, Eschman y Zuccolotto 2012), que permite llevar un registro exacto de los datos de rt y precisión ('accuracy', en inglés) vinculados a cada reactivo respondido por los estudiantes. Este fue instalado en los computadores del Laboratorio LATLA ${ }^{7}$ del Programa ELE-UdeC, lugar en que se realizaron habitualmente las clases a las que asistieron los estudiantes. La aplicación experimental fue administrada a todos los sujetos que pertenecían a un curso en forma simultánea por los docentes a cargo como una actividad normal de clases para resguardar la validez ecológica del estudio. A los estudiantes no se les permitió tener acceso al diccionario ni a correctores de texto durante el experimento; tampoco tuvieron la posibilidad de hacer preguntas, por ejemplo, sobre dudas respecto del léxico o la interpretación de las oraciones durante el desarrollo del experimento.

El diseño experimental consistió en la presentación aleatoria de todos los reactivos por pantalla a todos los sujetos. Cuando los participantes respondían correctamente, la aplicación emitía el mensaje '¡Correcto!' y luego se mostraba un nuevo reactivo. En caso de que la respuesta ingresada hubiese sido errónea, el experimento ejecutaba aleatoriamente tres posibles acciones: (1) se mostraba el estímulo siguiente sin entregar FCE. (2) Se entregaba un mensaje de FCMD, como por ejemplo, 'Necesitas usar una preposición que indique finalidad: para', y se presentaba simultáneamente el mismo estímulo para ser respondido nuevamente. (3) Se entregaba un mensaje de FCMI, como por ejemplo, 'Necesitas usar una preposición que indique finalidad', junto con la presentación del mismo estímulo para que el estudiante lo respondiese en segunda instancia. En los casos (2) y (3), si el estudiante se equivocaba nuevamente después de haber sido expuesto al tratamiento de FCE, el programa pasaba al reactivo siguiente.

7 LATLA: Laboratorio de Aplicaciones Tecnológicas en Lingüística Aplicada del Programa de Español como Lengua Extranjera, ELE-UdeC. 
Para el procesamiento de los datos utilizamos el programa E-DataAid incorporado al paquete E-Prime 2.0. El análisis fue realizado con el programa EXCEL del paquete Microsoft Office 2010 e incluyó la obtención de estadísticos descriptivos; tales como, porcentajes de efectividad, promedios de rt, diferencias de porcentaje de efectividad, diferencias de $\mathrm{rt}$ promedio, $\mathrm{rt}$ de procesamiento máximo y mínimo, y diferencias de rt máximo y mínimo; además de tablas y gráficos.

Previo al análisis de los rt promedio y datos de precisión de las instancias de respuesta a los estímulos, preparamos los datos siguiendo los procedimientos de varios estudios cognitivos dentro del campo de la Lingüística Aplicada: Jiang y Nekrasova 2007; Yamashita y Jiang 2010; Wolter y Gyllstad 2013; Wolter y Yamashita 2015. En primer lugar, se seleccionaron solo las respuestas que habían sido respondidas erróneamente en primera instancia, y que fueron respondidas correctamente en segunda instancia luego de recibir el tratamiento de FCE. En segundo lugar, para reducir la influencia de los datos atípicos que dificultasen observar con claridad las tendencias de rt, excluimos todas las respuestas correctas en segunda instancia que presentaban un nivel de corte de rt inferior a $350 \mathrm{~ms}$. y también los datos de aquellas respuestas correctas en segunda instancia cuyos $\mathrm{rt}$ superaban o eran inferiores a dos desviaciones estándares del rt promedio individual de cada participante, lo que significó una pérdida de $6,2 \%$ de los datos respondidos correctamente en segunda instancia. Este nivel se sitúa dentro de los rangos informados en los estudios señalados anteriormente, por ejemplo, Yamashita y Jiang 2010, y Wolter y Yamashita 2015.

\section{RESULTADOS Y DISCUSIÓN}

\subsection{HIPÓTESIS 1:}

El promedio de los rt en el procesamiento del FCMI será mayor que el del FCMD e irá acompañado de una menor efectividad del FCE en ambos niveles de competencia en un corto plazo

Al comparar en la Tabla 1 el promedio de rt y el porcentaje de efectividad inmediata asociados a los dos tipos de FCE estudiados en ambos niveles de competencia, se puede apreciar, en primer lugar, que el FCMD es procesado en menor rt (A2=1679,13 ms.; B1=1593,28 ms.; promedio=1636,21 
ms.) que el FCMI en ambos grupos de competencia (A2=2253,13 ms.; $\mathrm{B} 1=2157,72 \mathrm{~ms}$.; promedio $=2205,43 \mathrm{~ms}$.), respectivamente. Los menores rt del FCMD van acompañados de una mayor efectividad en ambos niveles de competencia ( $\mathrm{A} 2=91,3 \%$; $\mathrm{B} 1=91,8 ;$ promedio $=91,6 \%$ ), comparados con los del FCMI (A2=26,5\%; B1=36,7\%; promedio=31,6\%). Así también, en el FCMI se observa una mayor diferencia en la efectividad entre los grupos A2 y B1 (Diferencia B1-A2=10,2 puntos porcentuales) que la diferencia del FCMD (Diferencia B1-A2 $=0,5$ puntos porcentuales). Esto quiere decir que el desempeño de la efectividad del FCE es mucho más cercano entre los niveles A2 y B1 del FCMD, que la de dichos niveles en el FCMI. Esto indicaría que el FCMD tendería a promover un desempeño de efectividad relativamente similar entre ambos niveles de profiencia, en contraste con el FCMI que estimularía un desempeño más dispar entre ambos niveles, con una superioridad relativa de efectividad del nivel B1 por sobre la del nivel A2.

Tabla 1. Porcentaje de efectividad y promedio de rt por competencia y tipo de FCE

\begin{tabular}{llcccc}
\hline Nivel & Estadístico & FCMD & FCMI & Promedio & Diferencia \\
\hline \multirow{4}{*}{ A2 } & $\mathrm{N}^{\circ}$ errores tratados & 219 & 230 & 224,5 & -11 \\
& $\mathrm{~N}^{\circ}$ respuestas correctas & 200 & 61 & 130,5 & 139 \\
& \% efectividad & 91,3 & 26,5 & 58,9 & 64,8 \\
& rt promedio & 1679,13 & 2253,13 & 1966,13 & $-574,00$ \\
\hline \multirow{4}{*}{ B1 } & $\mathrm{N}^{\circ}$ errores tratados & 147 & 147 & 147 & 0 \\
& $\mathrm{~N}^{\circ}$ respuestas correctas & 135 & 54 & 94,5 & 81 \\
& \% efectividad & 91,8 & 36,7 & 64,3 & 55,1 \\
& rt promedio & 1593,28 & 2157,72 & 1875,50 & $-564,44$ \\
\hline \multirow{4}{*}{$\mathrm{N}^{\circ}$ total errores tratados } & 366 & 377 & & \\
& efectividad promedio & 91,6 & 31,6 & & \\
& Diferencia \% efectividad & $-0,5$ & $-10,2$ & & \\
& Promedio rt & 1636,21 & 2205,43 & & \\
& Diferencia rt & 85,85 & 95,41 & & \\
\hline
\end{tabular}

Al realizar la comparación de rt y efectividad de los dos niveles de competencia en ambos tipos de FCE, evidenciamos que tanto el FCMD y FCMI son procesados respectivamente en mayor $\mathrm{rt}$ en el nivel A2 $(\mathrm{FCMD}=1679,13 \mathrm{~ms}$.; $\mathrm{FCMI}=2253,12 \mathrm{~ms}$.; promedio $=1966,13 \mathrm{~ms}$.) que en el grupo B1 (FCMD=1593,28 ms.; FCMI=2157,72 ms.; promedio=1875,50 $\mathrm{ms}$.). Específicamente, se observa una mayor diferencia de rt promedio entre el FCMD y FCMI en el nivel A2 (Diferencia $=574,00 \mathrm{~ms}$.) que en el nivel $\mathrm{B} 1$ (Diferencia $=564,44 \mathrm{~ms}$.). 
En cuanto a la efectividad, el promedio de ambos tipos de FCE del nivel A2 (58,9\%) es menor que el de B1 (64,3\%). Por otra parte, la diferencia de efectividad entre el FCMD y el FCMI del nivel A2 es de 64,8 puntos porcentuales, superando la diferencia de efectividad entre el FCMD y el FCMI del nivel B1 (55,1 puntos porcentuales). Estos datos indicarían que con un mejoramiento en la competencia disminuirían tanto los rt de procesamiento del FCE en general, como las diferencias en los rt de procesamiento entre ambos tipos de FCE; por otra parte, también mejoraría la efectividad, con una menor diferencia de efectividad entre ambos tipos de FCE.

Para comprender de mejor manera el comportamiento de los dos tipos de FCE estudiados respecto de la competencia, adicionalmente analizamos los $\mathrm{rt}$ de procesamiento del FCE máximos y mínimos de los participantes acorde al tipo de FCE y por nivel de competencia. De esta forma, complementariamente a lo que pudimos observar anteriormente en la Tabla 1, que aunque el rt promedio de ambos tipos de FCE es mayor en A2 (1966,13 ms.) que en B1 (1875,50 ms.), en la Tabla 2 obtenemos que en $\mathrm{B} 1$ hay una mayor diferencia $(1807,22 \mathrm{~ms}$.) entre la diferencia rt máxmín del FCMD y el FCMI que en el nivel A2 (882,17 ms.). Por otra parte, mirado desde la perspectiva del nivel lingüístico, el grupo B1 casi dobla la diferencia de rt máximo-mínimo de A2, tanto en el FCMD (1404,04 ms.) como en el FCMI (2329,09 ms.).

Tabla 2. Rt máximos y mínimos según el nivel de competencia y tipo de FCE

\begin{tabular}{lccc}
\hline Rt máximo y mínimo & A2 & B1 & $\begin{array}{c}\text { Diferencia rt nivel } \\
\text { competencia }\end{array}$ \\
\hline Máximo FCMD & 2532,29 & 3734,33 & \\
Mínimo FCMD & 969,80 & 767,80 & \\
\hline \multicolumn{1}{c}{ Diferencia rt máx-mín } & 1562,49 & 2966,53 & $-1404,04$ \\
\hline Máximo FCMI & 3673,33 & 5785,50 & \\
Mínimo FCMI & 1228,67 & $1.011,75$ & \\
\hline \multicolumn{1}{c}{ Diferencia rt máx-mín } & 2444,66 & 4773,75 & $-2329,09$ \\
\hline Diferencia rt tipo FCE & 882,17 & 1807,22 & \\
\hline
\end{tabular}

Estos datos apuntarían, en primer lugar, a una mayor variabilidad intersujeto en los rt de procesamiento en el nivel B1 comparados con los del nivel A2, es decir, una mayor amplitud en los rangos máximos y mínimos en ambos tipos de FCE en el nivel B1. Esto se podría deber al hecho de que un mejoramiento en el nivel de competencia implica un mayor repertorio de las variables que definen el uso preposicional correcto, lo cual mostraría un 
mayor efecto asociado a las diferencias individuales. Esto se traduciría en mayores diferencias en los tiempos de análisis y reflexión de las posibilidades correctas estimuladas por el FCE dentro del nivel B1, y en menor grado en el nivel A2. En segundo lugar, los datos serían también indicativos de una mayor variabilidad intersujeto en el procesamiento del FCMI comparado con el FCMD, con una mayor amplitud en los rangos máximos y mínimos en FCMI en ambos niveles de competencia. Respecto de esto último, creemos que estas diferencias estarían asociadas a los rasgos inherentes al FCMI, cuya aplicación implica una mayor ambigüedad en la estimulación de los factores que definen la corrección preposicional y que conlleva a una mayor permeabilidad a las diferencias individuales en el procesamiento del FCMI.

\subsection{HIPÓTESIS 2:}

El promedio de los rt en el procesamiento del FCE será mayor en las preposiciones que presentan mayor número de errores que en las preposiciones menos problemáticas

De acuerdo con los datos presentados en la Tabla 3 , en primer lugar, podemos visualizar un patrón de efectividad del FCE bastante claro respecto de la competencia (Gráfico 1); esta es mayor en B1 que en A2 en todas las preposiciones. En segundo lugar, en cuanto al rt promedio de procesamiento del FCE por preposición (Gráfico 2), como es esperable, obtenemos que este es mayor en 3 preposiciones del nivel A2 comparado con el $\mathrm{rt}$ del nivel B1; específicamente, de mayor a menor, el rt en A2 es mayor en con (A2=2160,00 ms.; B1=1813,61 ms.), por (A2=1901,63 ms.; B1=1680,07 ms.) y de (A2=1658,68 ms.; B1=1365,60 ms.). De estos tres hallazgos, el mayor rt del nivel A2 comparado con el nivel B1 de la preposición por coincide con el reportado por Ferreira, Oportus y Fuentes (2016). Por otra parte, el nivel B1 presenta un rt mayor que el de $\mathrm{A} 2$ en para $(\mathrm{B} 1=2408,72$ ms.; A2=2212,67 ms.), y en la preposición $a(B 1=1535,10$ ms.; A2 $=1308,89$ $\mathrm{ms}$.), estos dos últimos hallazgos también coincidentes por lo informado por Ferreira (ibíd.) 
Tabla 3. Rt promedio y porcentaje efectividad según competencia y preposición

\begin{tabular}{|c|c|c|c|c|c|c|}
\hline Nivel & 1 Estadístico & $\mathrm{a}$ & de & con & para & por \\
\hline \multirow{4}{*}{ A2 } & $\mathrm{N}^{\circ}$ errores tratados & 115 & 84 & 29 & 118 & 103 \\
\hline & $\mathrm{N}^{\circ} \mathrm{re}$ & 63 & 44 & 16 & 67 & 71 \\
\hline & $\%$ ef & 54,8 & 52,4 & 55,2 & & 68,9 \\
\hline & Rt promedio & $1.308,89$ & $1.658,68$ & 2160,00 & 2212,67 & 1901,63 \\
\hline \multirow{4}{*}{ B1 } & $\mathrm{N}^{\circ}$ errores tratados & 78 & 64 & 37 & 64 & 51 \\
\hline & $\mathrm{N}^{\circ} \mathrm{re}$ & 49 & 35 & 28 & 39 & 38 \\
\hline & $\% \mathrm{e}$ & 62, & 54, & 75 & 60,9 & 74,5 \\
\hline & Rt promedio & 1535,10 & 1365,60 & 1813,61 & 2408,72 & 1680,87 \\
\hline & $N^{\circ}$ tot: & & & 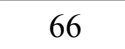 & 18 & 1 \\
\hline & Efectividad pro & 58, & 53 , & 65,4 & 58,9 & 71,7 \\
\hline \multicolumn{2}{|r|}{ Diferencia $\%$ efectividad } & $-8,0$ & $-2,3$ & $-20,5$ & $-4,1$ & $-5,6$ \\
\hline \multirow{2}{*}{\multicolumn{2}{|c|}{$\begin{array}{l}\text { Promedio rt } \\
\text { Diferencia rt }\end{array}$}} & 1422,00 & 1512,14 & 1986,81 & 2310,70 & 1791,25 \\
\hline & & $-226,21$ & 293,08 & 346,39 & $-196,05$ & 220,76 \\
\hline
\end{tabular}

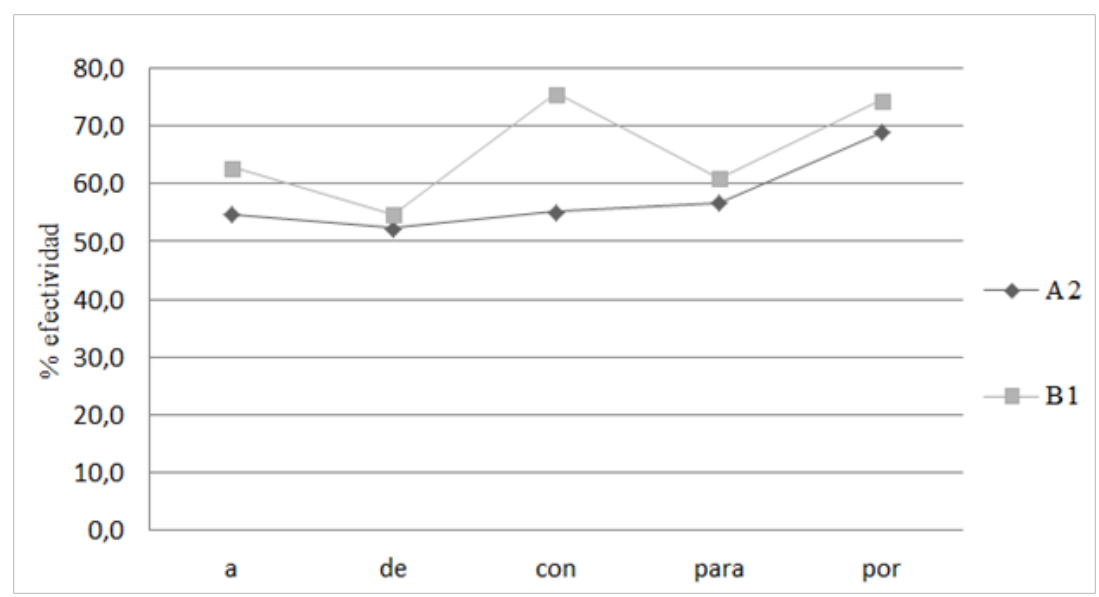

Gráfico 1. Efectividad promedio por competencia y preposición 


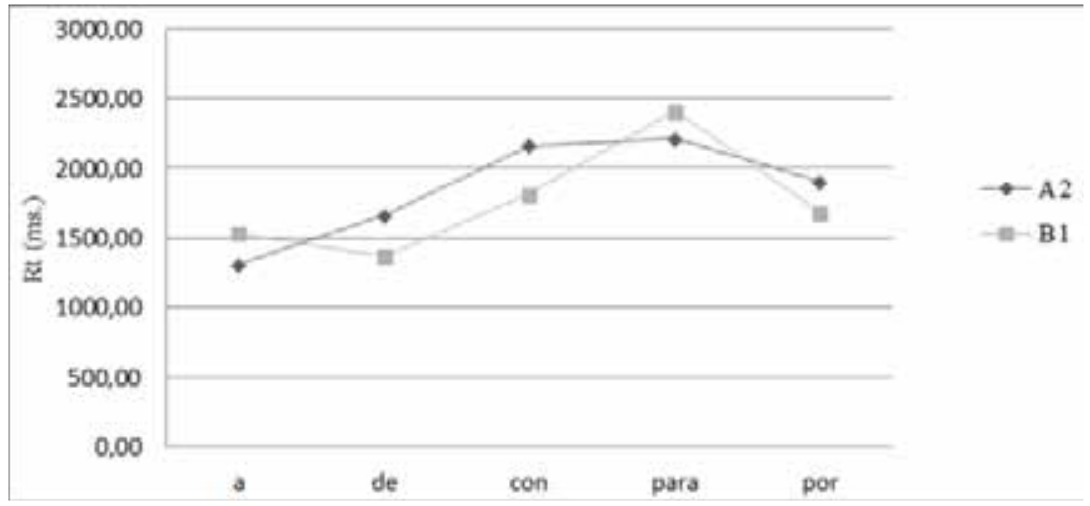

Gráfico 2. Rt promedio por competencia y preposición

Con los antecedentes señalados, se determinó analizar el rt y la efectividad del FCE de cada preposición para dar cuenta del comportamiento preposicional individual. Examinamos las preposiciones siguiendo el orden de acuerdo a la dificultad que presentaron a los participantes, es decir, acorde el número total de errores tratados en el experimento en orden descendente; estos fueron los siguientes: a (193), para (182), por (154), de (148) y con (66). Respecto de lo anterior, observamos que el ordenamiento acorde a este criterio reproduce íntegramente el orden de dificultad dado por Ferreira y Lafleur (2015). Así también, la preposición $a$, con el mayor número de errores en el presente estudio, muestra la mayor frecuencia de errores preposicionales informadas por otros autores (Fernández López 1994; Giraldo 1997; Campillos 2014).

En efecto, al realizar un análisis comparativo más detallado por preposición, y comenzando por la preposición $a$, vemos que en el nivel A2 esta muestra el segundo número más alto de errores tratados (115), mientras que en el grupo B1 alcanza el número más alto (78). Por su parte, la efectividad del FCE es mayor en B1 (62,8\%) que en A2 (54,5\%), y así también, el rt asociado a esta efectividad es mayor en B1 (1535,10 ms.) que en A2 (1308,89 ms.). De acuerdo con lo anterior, tomando en cuenta el número de errores tratados y los rt de procesamiento del FCE en ambos niveles, $a$ se presenta como una preposición de dificultad importante en ambos niveles, con una complejidad de procesamiento de rt persistente en el nivel B1. Estos datos apuntan en la dirección señalada por estudios previos respecto de la dificultad de adquisición que presenta esta preposición debido a su alta frecuencia de uso y múltiples significados y funciones que puede adoptar (Fernández López 1994; Giraldo 1997; Campillos 2014; Ferreira, Elejalde y Vine 2014; Ferreira y Lafleur 2015). 
De las cinco preposiciones estudiadas, para muestra el mayor $\mathrm{rt}$ de procesamiento del FCE en ambos grupos de competencia, con un rt mayor en el grupo B1 (A2 =2212,67 ms.; B1=2408,72 ms.), dato coincidente con Ferreira, Oportus y Fuentes (2016). Así, también, para es la preposición que muestra un rt de procesamiento más cercano entre A2 y B1, con la menor diferencia entre ambos niveles (196,05 ms.). Respecto de la efectividad, en el nivel A2, para presenta la segunda mayor efectividad (56,8\%), muy cercana al nivel de efectividad que alcanzan $a$, de y con; mientras que en el nivel B1, esta alcanza la cuarta efectividad $(60,9 \%)$. La diferencia de la efectividad entre ambos grupos es la segunda más cercana (4,1 puntos porcentuales) después de la preposición de (2,3 puntos porcentuales). Los datos anteriores sobre el $\mathrm{rt}$ de procesamiento nos llevan a reflexionar que la corrección de los errores de para es una de la más complejas para los estudiantes de ambos niveles en este estudio. No obstante, aunque en el nivel A2 los estudiantes toman más tiempo en procesar el FCE comparado con otras preposiciones, su efectividad de $56,8 \%$ es levemente superior a la de las preposiciones $a$, de y con; lo que no sucede en el nivel B1, en donde el rt es el más alto y la efectividad del FCE es menor que la de con, por y $a$. Estos últimos datos indicarían que para presenta una importante dificultad en la adquisición de sus usos correctos, con una complejidad persistente manifiesta en el rt de procesamiento, más evidente en el nivel B1 que en el nivel A2.

El uso de la preposición por es una problemática importante en el nivel A 2 con 103 errores tratados, los que disminuyen en casi 50\% en B1 (51 errores tratados). También se observa un descenso en el rt de procesamiento del FCE acorde la competencia, desde 1901,63 ms. en A2 hasta 1680,87 ms. en el nivel B1. De esta forma, podemos evidenciar que aunque el $\mathrm{rt}$ de $p o r$ es el tercero más alto en ambos grupos (A2=1901,63 ms.; B1=1680,87 ms.; promedio $=1791,25 \mathrm{~ms}$.), el rt disminuye en $220,76 \mathrm{~ms}$ en $\mathrm{B} 1$ respecto del nivel A2. Por otra parte, la efectividad mejora en B1 (74,5\%), comparado con el grupo A2 (68,9\%). Estos datos nos inducen a sostener que por es una preposición que muestra un comportamiento esperado de efectividad y de procesamiento acorde con el mejoramiento de la competencia, es decir, disminución de los errores y rt del FCE en el nivel B1. De acuerdo a lo anterior, la adquisición y complejidad cognitiva vinculada al procesamiento de los errores de la preposición por durante el tratamiento de FCE mejorarían sustantivamente con el avance de la competencia.

Al igual que en Ferreira, Oportus y Fuentes (2016) y Fernández (1994), la preposición de presenta una dificultad media relativas a los participantes, con 84 y 64 errores tratados en A2 y B1, respectivamente. No obstante, su uso correcto sería de naturaleza más bien compleja puesto que el porcentaje de efectividad del FCE es el más bajo en ambos grupos de competencia 
del estudio (A2=52,4\%; B1=54,7\%). Asimismo, podemos apreciar que en el nivel B1 la efectividad del FCE va acompañada de una disminución importante del rt de procesamiento (segunda mayor diferencia entre A2 y B1, correspondiente a 293,08 ms). Esto nos lleva a plantear que la dificultad y complejidad de esta preposición tienden a mejorar gradualmente con el avance de la competencia, lo que puede apreciarse en la reducción del rt del procesamiento FCE y mejoramiento de la efectividad en el grupo B1.

Finalmente, en lo que respecta a la preposición con, esta representaría la menor dificultad en ambos niveles en tanto que muestra el menor número de errores tratados $(\mathrm{A} 2=29 ; \mathrm{B} 1=37)$. No obstante, algunos de los valores incluidos en el experimento (compañía de personas, instrumento, unión de cosas) resultarían complejos, a la luz que los rt de procesamiento promedio del FCE en los niveles A2 (2160,00 ms.) y B1 (1813,61 ms.) alcanzan los segundos rt más altos en ambos niveles. Por otra parte, esta preposición muestra la mayor diferencia en los $\mathrm{rt}$ de procesamiento $(346,39 \mathrm{~ms}$.) y la mayor diferencia en efectividad (20,5 puntos porcentuales) entre ambos niveles, con una importante disminución en el rt de procesamiento del FCE y mejoramiento en la efectividad en el nivel B1 como resultado del mejor nivel de competencia del grupo B1. De acuerdo a estos datos, con seguiría un patrón esperado al nivel de competencia, con un mejoramiento de la efectividad y disminución de los rt de procesamiento consistentes en el nivel B1.

De acuerdo con la Tabla 4, al examinar por separado el comportamiento del rt y la efectividad de los dos tipos de FCE con respecto a la competencia y a lo largo de las preposiciones estudiadas, podemos apreciar con claridad que el rt de procesamiento del FCMI es mayor que el del FCMD en todas las preposiciones respectivas en el Gráfico 3, con una efectividad inferior del FCMI a la del FCMD en todas ellas (Gráfico 4). Específicamente, al analizar el FCMD acorde la competencia, partiendo por el nivel A2, observamos que el rt de procesamiento por preposición, de mayor a menor, es para $(2052,13$ ms.), por (1788,67 ms.), con (1764,78 ms.), de (1535,66 ms.) у a (1278,04 ms.). En cuanto al nivel B1, el rt, ordenado de mayor a menor es: para (2270,75 ms.), por (1562,68 ms.), a (1436,06 ms.), de (1342,47 ms.), у con (1272,33 ms.). Según lo anterior, se observa una coincidencia en los dos rt mayores del FCMD en ambos grupos de competencia, para y por. Asimismo, también podemos ver que el $\mathrm{rt}$ de las preposiciones de, con, y por es mayor en A2 que en B1; mientras que para y $a$ obtienen un $\mathrm{rt}$ mayor en el nivel $\mathrm{B} 1$ que en A2. De acuerdo con estos datos, sobresale que el mejoramiento en la competencia del nivel B1 respecto del tratamiento de errores de para y $a$ con FCMD aparece asociado a un mayor rt para el nivel B1. Esto daría cuenta de la complejidad evidente implicada en la resolución de los errores de estas dos preposiciones cuando son tratados con FCMD en el nivel B1. 
Tabla 4. Porcentaje efectividad y rt promedio por competencia, tipo de FCE y preposición

\begin{tabular}{cccccccc}
\hline $\begin{array}{c}\text { Tipo } \\
\text { FCE }\end{array}$ & Nivel & Estadístico & a & de & con & para & por \\
\hline & A2 & Rt promedio & 1278,04 & 1535,66 & 1764,78 & 2052,13 & 1788,67 \\
& A2 & $\mathrm{N}^{\circ}$ errores & 57 & 41 & 9 & 59 & 53 \\
& A2 & $\mathrm{N}^{\circ}$ corregidos & 51 & 38 & 9 & 53 & 49 \\
FCMD & A2 & \% efectividad & 89,5 & 92,7 & 100 & 89,8 & 92,5 \\
\cline { 2 - 8 } & B1 & Rt promedio & 1436,06 & 1342,47 & 1272,23 & 2270,75 & 1562,68 \\
B1 & $\mathrm{N}^{\circ}$ errores & 38 & 36 & 14 & 33 & 26 \\
B1 & $\mathrm{N}^{\circ}$ corregidos & 35 & 33 & 13 & 28 & 25 \\
B1 & \% efectividad & 92,1 & 94,4 & 92,9 & 84,8 & 96,2 \\
\hline A2 & Rt promedio & 1440,00 & 2437,83 & 2668,14 & 2820,43 & 2153,23 \\
A2 & $\mathrm{N}^{\circ}$ errores & 58 & 43 & 20 & 59 & 50 \\
A2 & $\mathrm{N}^{\circ}$ corregidos & 12 & 6 & 7 & 14 & 22 \\
FCMI & $\%$ efectividad & 20,7 & 14,0 & 35,0 & 23,7 & 44,0 \\
\hline & B1 & Rt promedio & 1782,71 & 2152,00 & 2282,80 & 2759,91 & 1908,15 \\
B1 & $\mathrm{N}^{\circ}$ errores & 40 & 28 & 23 & 31 & 25 \\
B1 & $\mathrm{N}^{\circ}$ corregidos & 14 & 1 & 15 & 11 & 13 \\
B1 & $\%$ efectividad & 35,0 & 3,6 & 65,2 & 35,5 & 52,0 \\
\hline
\end{tabular}

Continuando con el análisis del FCMD, pero enfocados en un análisis individual del $\mathrm{rt}$ de procesamiento y efectividad por preposición ${ }^{8}$, nos encontramos con patrones de comportamiento más dispares, como se observa en los Gráficos 3 y 4 . La preposición para, que exhibe los mayores rt en ambos grupos, muestra bajos niveles relativos de efectividad del FCE. En A2 la efectividad del FCMD es la segunda más baja (89,9\%); mientras que en el grupo B1, la efectividad del FCMD es la más baja entre las cinco preposiciones $(84,8 \%)$. Por su parte, la preposición por, con el segundo $\mathrm{rt}$ más alto de procesamiento del FCMD en ambos niveles, muestra una alta efectividad relativa del FCMD al compararla con las otras preposiciones. Por ejemplo, en el nivel A2, la efectividad en la corrección de los errores de por se ubica en el segundo lugar (92,5\%); mientras que en el nivel B1, la efectividad del FCMD es la más alta (96,2\%). A diferencia de por y para, la preposición $a$ muestra bajos niveles de rt de procesamiento y bajos niveles de efectividad. En el caso de A2, esta muestra el menor rt (1278,04 ms.) con el menor nivel de efectividad del FCMD $(89,5 \%)$, mientras que en el caso de

\footnotetext{
8 Se dejó fuera la preposición CON debido al relativamente bajo número de errores tratados en el experimento ( $\mathrm{A} 2=9$ errores y $\mathrm{B} 1=14$ errores).
} 
B1, $a$ muestra el tercer lugar de rt (1436,06 ms.), con el segundo nivel más bajo de efectividad $(92,1 \%)$. Por último, de presenta niveles relativamente bajos de rt de procesamiento del FCMD, con el cuarto lugar en ambos grupos (A2=1535,66 ms.; B1=1342,47 ms.), con una efectividad relativamente alta en ambos niveles (A2=92,7\%; B1 (94,4\%).

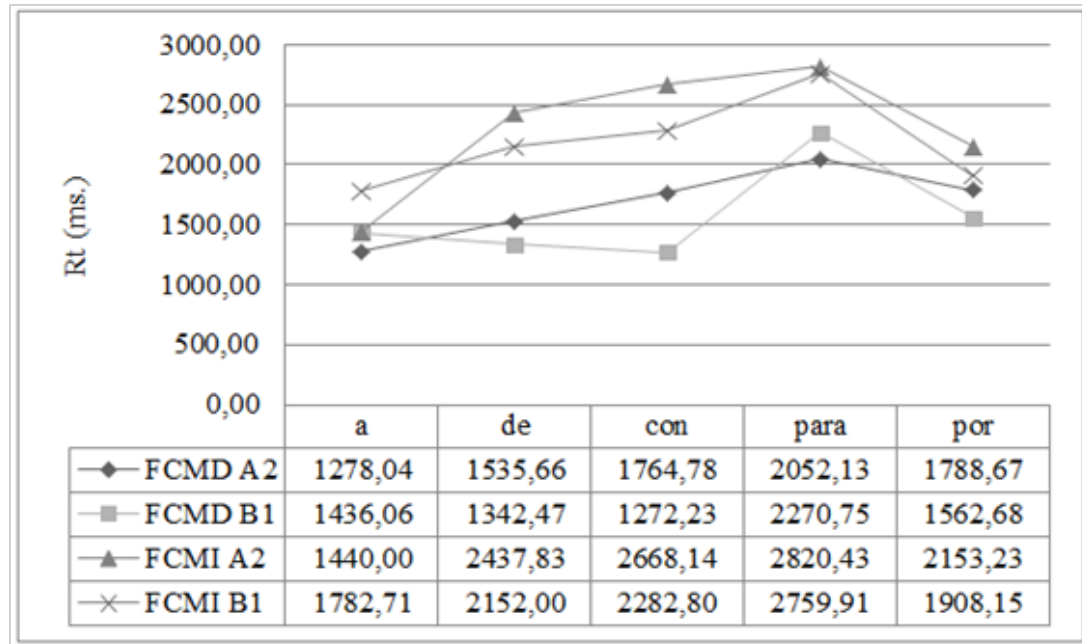

Gráfico 3. Promedio de rt por tipo de FCE, competencia y preposición

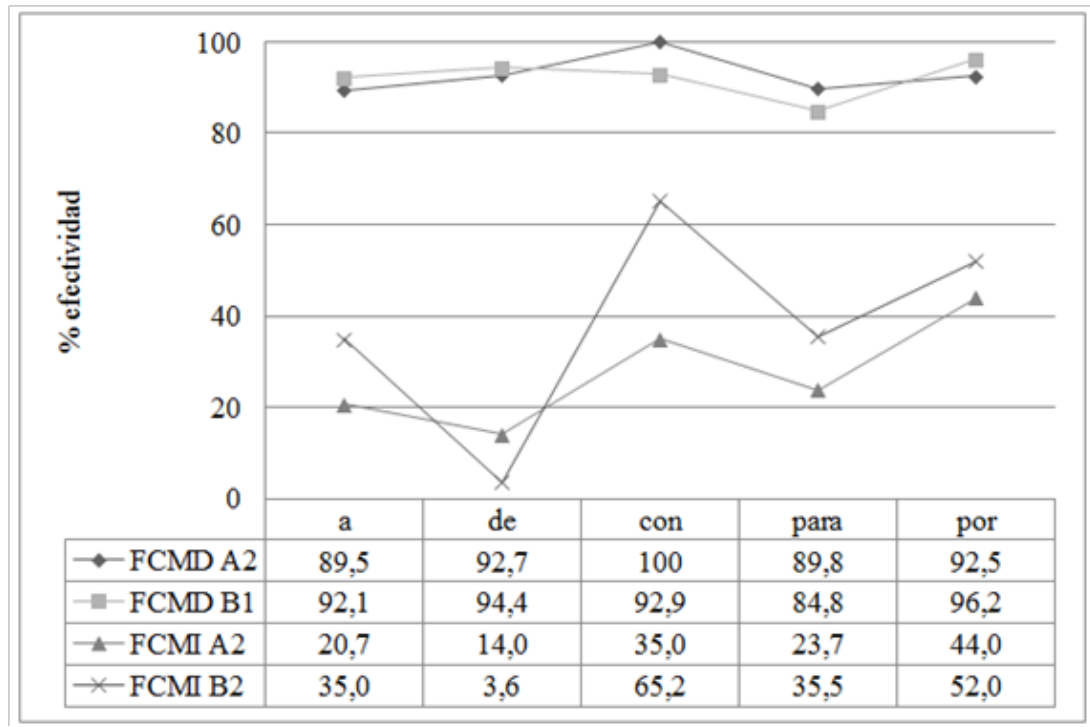

Gráfico 4. Porcentaje efectividad por tipo de FCE, competencia y preposición 
En la modalidad de FCMI se observan algunas tendencias bastante claras en la Tabla 4 y los gráficos 3 y 4 . En primer lugar, respecto del rt, ambos grupos de competencia siguen el mismo ordenamiento de mayor a menor: para (A2=2820,43 ms.; B1=2759,91 ms.), con (A2=2668,14 ms.; B1=2282,80 ms.), de (A2=2437,83 ms.; $\mathrm{B} 1=2152,00 \mathrm{~ms}$ ), por (A2=2153,23 ms.; $\mathrm{B} 1=1782,71)$, y $a(\mathrm{~A} 2=1440,00 ; \mathrm{B} 1=1782,71 \mathrm{~ms}$.). Luego, al comparar el rt de procesamiento de cada preposición respectiva por nivel, vemos que el $\mathrm{rt}$ del nivel A2 supera al del nivel B1 en todas ellas, exceptuando la preposición $a$. En contraste, al examinar la efectividad del FCMI, encontramos que la efectividad del grupo B1 supera en todas las preposiciones al nivel A2, exceptuando la preposición de. Estos datos nos dan indicio para plantear que el FCMI sigue un comportamiento más cercano que el del FCMD en lo que esperaríamos respecto de la competencia en la mayor parte de las preposiciones estudiadas. Es decir, el nivel A2 requiere un mayor tiempo de procesamiento del FCE que el grupo B1, y a su vez, obtiene menor efectividad que el grupo de nivel B1. De aquí se desprende que el FCMI, en razón de sus rasgos propios, pareciera ser más adecuado para resolver los problemas preposicionales del nivel $\mathrm{B} 1$ que en un nivel con menor desarrollo lingüístico, como es el A2.

En el análisis individual de la efectividad del FCMI por preposición y respecto de los rt, tenemos que la preposición para, que requiere el mayor $\mathrm{rt}$ de procesamiento en ambos grupos, obtiene una efectividad relativamente baja en ambos niveles, con el tercer lugar de efectividad en A2 $(23,7 \%)$ y B1 $(35,5 \%)$. En el caso de con, esta preposición presenta el segundo rt de procesamiento del FCMI en ambos grupos de competencia y obtiene el segundo lugar de efectividad en A2 y el primero en B1. De acuerdo a ello, esta preposición, aunque en base a un número reducido de errores tratados $(\mathrm{A} 2=20 ; \mathrm{B}=23$ ) conllevaría cierta complejidad para los estudiantes puesto que necesitan un rt considerable de procesamiento, pero con la ayuda del FCMI pueden resolver el problema preposicional logrando una efectividad comparada con las de las otras preposiciones relativamente alta $(\mathrm{A} 2=35,0$ $\%, \mathrm{~B} 1=65,2 \%)$. Surgiría entonces como un aspecto interesante de explorar a futuro el origen de esta complejidad explorando más en detalle el comportamiento de los tres usos lingüísticos específicos incluidos en el experimento: los valores generales de compañía, unión de cosas e instrumento expresados por dicha preposición. La preposición de, con el tercer lugar de rt de procesamiento en ambos grupos, muestra una complejidad media relativa. No obstante, sus errores resultan considerablemente difíciles de resolver con la ayuda del FCMI en ambos grupos de competencia (A2=14,0\%; B1 =3,6\%). Continuando con el cuarto lugar de rt en ambos grupos, por muestra la mayor efectividad del FCMI en A2 (44,0\%) y el segundo lugar en B1 (52,0\%). Esta 
evidencia apunta a que esta preposición presenta una complejidad cognitiva relativamente baja para los estudiantes, pues con la ayuda ofrecida por el FCMI pueden resolver dichos errores con una efectividad relativamente alta en ambos niveles. En último lugar, la preposición $a$, que exhibe el menor rt de procesamiento del FCMI, por lo que tendría una baja complejidad cognitiva, muestra la segunda efectividad más baja en ambos niveles (A2 $=20,7 \%$; $\mathrm{B} 1=35,5 \%$ ). Por ello, el FCMI resultaría relativamente poco eficiente para resolver los errores de la preposición A en ambos niveles de competencia.

\section{CONCLUSIONES}

El objetivo de este estudio ha sido examinar empíricamente el $\mathrm{rt}$ de procesamiento y la efectividad del FCMI, comparado con el FCMD durante el tratamiento de los errores preposicionales de $a$, de, por, para, en y con en aprendientes de ELE de nivel A2 y B1. Para ello identificamos: (1) la dificultad preposicional representada por la frecuencia y recurrencia de errores que las afectan; (2) la complejidad lingüística preposicional referida a la naturaleza de los valores, significados y usos asignados a cada preposición en la L2, y (3) la complejidad cognitiva correspondiente al tiempo de procesamiento empleado en la resolución de un problema preposicional tratado con una modalidad de FCE.

Entre los hallazgos más interesantes, encontramos que en el tratamiento de los errores preposicionales estudiados, el FCMI es el que genera mayor $\mathrm{rt}$ de procesamiento tanto en el nivel A2, como en el nivel B1. Esto concuerda con los planteamientos teóricos, en cuanto a que el FCMI, comparado con el FCMD, conllevaría un grado mayor de ambigüedad en el mensaje correctivo debido a su naturaleza inherente, lo que estimularía una mayor reflexión metalingüística para resolver el error. No obstante, el FCMI también presenta una menor efectividad en el corto plazo que la del FCMD en el contexto del diseño experimental utilizado. De esta forma, sería interesante examinar a futuro si el mayor rt del FCMI, asociado a un mayor nivel de elaboración cognitiva que el del FCMD, difiere del FCMD en términos de estimular una mayor o menor efectividad a largo plazo.

Asimismo, observamos una mayor dispersión en los rt de procesamiento del FCMI que en el FCMD, y también una mayor dispersión en el rt de procesamiento del FCMI en el grupo B1 que en el nivel A2. De esta forma, el FCMI suscitaría una mayor influencia de las diferencias individuales 
en el procesamiento del FCE que la del FCMD, y esta sería mayor en el nivel B1 que en el nivel A2. En este sentido, nuestra muestra incluyó seis orígenes lingüísticos distintos que explicarían estas variaciones. Resultaría también interesante investigar en una muestra más representativa de lenguas distintas, la influencia que ejerce la $\mathrm{L} 1$ en el rt de procesamiento del FCE en el contexto de ELE.

En cuanto al $\mathrm{rt}$ de procesamiento del FCMI durante el tratamiento de los errores individuales por preposición, los resultados muestran que la mayor complejidad cognitiva en ambos niveles de competencia se presenta en el siguiente orden, de mayor a menor, en las preposiciones para, con, de, por y $a$. Así también, el rt de procesamiento del FCMI es mayor en el nivel B1 que en el grupo A2 en todas las preposiciones, con la excepción de $a$, con una mayor efectividad en el corto plazo en el nivel B1 que en A2. Por ello, el FCMI parecería ser más efectivo para el tratamiento de errores preposicionales en el nivel B1 que en el nivel A2.

Finalmente, de acuerdo a los resultados obtenidos en este estudio experimental, podemos señalar que el rt de procesamiento y la efectividad de corto plazo del FCE parecen depender de la interrelación compleja de varios factores, entre ellos, la complejidad lingüística inherente a cada preposición, los valores y significados específicos de cada preposición seleccionados para el experimento, el tipo de FCE, el nivel de competencia, y la L1 de los participantes.

\section{REFERENCIAS BIBLIOGRÁFICAS}

Bitchener, John y Dana Ferris. 2012. Written corrective feedback in second language acquisition and writing. New York: Routledge.

Campillos Llanos, Leonardo. 2014. Las preposiciones en el habla no nativa de nivel intermedio: análisis de la interlengua basado en corpus. Revista Nebrija de Lingüística Aplicada 16(1): 5-27.

CONSEJO DE EUROPA. 2001. Marco común europeo de referencia para las lenguas: aprendizaje, enseñanza, evaluación. Consejo de Europa, Estrasburgo. [en línea]. Disponible en http:// cvc.cervantes.es/ensenanza/biblioteca_ele/marco/ [Consulta 27/10/2017]

Elejalde Gómez, Jessica y Anita Ferreira Cabrera. 2016. Errores de transferencia en comunidades de aprendizaje en línea por aprendientes de español como lengua extranjera. Trabalhos em Lingüistica Aplicada 55(3): 619-650.

ElLis, Rod. 2008. A Typology of Written Corrective Feedback Types. ELT Journal 63: 97-107.

FERnÁNDEZ LóPEZ, Sonsoles. 1994. Las preposiciones en la interlengua de aprendices de ELE. En J. Sánchez e I. Santos, I. (eds.). Problemas y Métodos en la Enseñanza del Español como Lengua Extranjera. Actas del IV Congreso Internacional de ASELE, pp. 367-380. Madrid: ASELE. 
Ferreira Cabrera, Anita. 2016. Prueba de Multinivel con Fines Específicos Académicos, Programa de Español como Lengua Extranjera ELE-UdeC, Universidad de Concepción. 2017. El efecto del feedback correctivo para mejorar la destreza escrita en ELE. Colombian Applied Linguistics Journal 19(1): 37-50.

Ferreira Cabrera, Anita y Jessica Elejalde Gómez. 2017. Análisis de errores recurrentes en el Corpus de Aprendices de Español como Lengua Extranjera, CAELE. Revista Brasileira de Lingüística Aplicada 50(1): 139-160

Ferreira Cabrera, Anita y Nahum Lafleur. 2015. Analyse et description des erreurs prépositionnnelles les plus fréquentes en espagnol L2. Lingüística y Literatura, Antioquia 68: 57-79.

Ferreira Cabrera, Anita; Jessica Elejalde Gómez y Ana Vine Jara. 2014. Análisis de errores asistido por computador basado en un corpus de aprendientes de español como lengua extranjera. Revista Signos 47(86): 385-411.

Ferreira Cabrera, Anita; René Oportus Torres y Karina Fuentes Riffo. 2016. Tiempos de respuesta y feedback correctivo escrito en aprendientes de ELE. Revue Francaise de Linguistique Appliquée 12(2): 109-122.

FERris, DANA. 2010. Second language writing research and written corrective feedback in SLA intersections and practical applications. Studies in Second Language Acquisition 32, special issue 02: 181-201.

Garzón Osorio, Amelia. (2012). Las preposiciones en composiciones de alumnos de VG1 y VG2. Un análisis de errores. (Tesis de Magíster no publicada). Universitetet i Oslo, Institutt for litteratur, områdestudier og europeiske språk, Det humanistiske fakultet.

Giraldo Silverio, Inocencio. 1997. Adquisición y uso correcto de las preposiciones a, en, para y por en niveles superiores. ASELE, Actas VIII, pp. 379-386.

JIANG, NAN. 2012. Conducting reaction time research in second language studies. New York: Routledge.

Jiang, Nan y Tatiana Nekrasova. 2007. The processing of formulaic sequences by second language speakers. The Modern Language Journal. 91(3): 433-445.

Lafleur, Nahum y Anita Ferreira Cabrera. 2016. Efectividad del feedback correctivo escrito directo para mejorar el aprendizaje de las preposiciones por y para en español / L2. Alpha 43: 57-74.

LEONTARIDI, ELENA. 2010. El uso del paradigma preposicional español por grecófonos desde el punto de vista morfológico, sintáctico y semántico. IX Congreso Internacional de Lingüística General. Actas del Congreso, pp. 1338-1356. [en línea] Disponible en: https:// www.academia.edu/1175492/_El_uso_del_paradigma_preposicional espa\%C3\%B1ol por_grec\%C3\%B3fonos_desde_ê__punto_de_vista_morfor $\%$ C $3 \%$ B 3 gico_ sint $\%$ C3\%A1ctico_y_sem\% $\%$ C3\%A1ntico_. [Consulta 27/10/2017].

LoNG, MichaEL. 1991. Focus on form: a design feature in language teaching methodology. En K.de Bot, R. Ginhsberg y C. Kramsch (eds.). Foreign language research in cross-cultural perspective, pp. 39-52. Amsterdam: John Benjamins.

Perea, Francisco. 2007. Análisis de errores y enseñanza de las preposiciones de la lengua española como LE. . MarcoELE, 5. [en línea] Disponible en: http://marcoele.com/ descargas/5/perea-preposiciones.pdf. [Consulta 27/10/2017].

Pérez Cino, WALDo. 2000. Manual práctico de la preposición española. Madrid: Editorial Verbum. S. L.

Regueiro Rodríguez, María Luisa. 2014. El desafío preposicional en ELE: A propósito de "Las preposiciones en el habla no nativa de nivel intermedio: análisis de la interlengua basado en corpus" de Leonardo Campillos Llanos. Revista Nebrija de Lingüistica Aplicada 16(6): 61-70. 
Serrano Alonso, Andrea. 2016. Las preposiciones en español: problemas en estudiantes de L2/LE, con especial atención a por y para. Trabajo de Fin de Grado. Universidad de La Rioja. Servicio de Publicaciones. [en línea] Disponible en: https://biblioteca.unirioja.es/ tfe_e/TFE001231.pdf [Consulta 27/10/2017]

Schneider, Walter, Amy Eschman y Anthony Zuccolotto. 2012. E-Prime User's Guide. Pittsburgh: Psychology Software Tools, Inc.

SHeEn, Younghee. (2011). Corrective Feedback Individual Differences and Second Language Learning. New York: Springer.

Williams, Jessica y JaCQueline Evans. 2009. ¿Qué clase de atención y a qué formas? En Catherine Doughty y Jessica Williams. Atención a la forma en la adquisición de segundas lenguas, pp. 153-170. Madrid: Editorial Edinumen.

Wolter, Brent y Henrik Gyllstad. 2013. Frequency of input and L2 collocational processing. Studies in Second Language Acquisition 35: 451-482.

Wolter, Brent y Junko Yamashita. 2015. Processing collocations in a second language: A case of first language activation? Applied Psycholinguistics 36: 1193-1221.

YAMASHITA, JUNKO Y NAN JiANG. 2010. L1 Influence on the acquisition of L2 collocations: Japanese ESL users and EFL learners acquiring English collocations. Tesol Quarterly 44(4): 647-668. 